\title{
Universality of transport properties in equilibrium, Goldstone theorem and chiral anomaly
}

\author{
Anton Yu. Alekseev*, Vadim V. Cheianov*, Jürg Fröhlich ${ }^{\dagger}$ \\ * Institute for Theoretical Physics, Uppsala University, Box 803, S-75108, Uppsala, Sweden \\ † Institut für Theoretische Physik, ETH-Hönggerberg, CH-8093, Zürich, Switzerland
}

(March 1998)

\begin{abstract}
We study transport in a class of physical systems possessing two conserved chiral charges. We describe a relation between universality of transport properties of such systems and the chiral anomaly. We show that the non-vanishing of a current expectation value implies the presence of gapless modes, in analogy to the Goldstone theorem. Our main tool is a new formula expressing currents in terms of anomalous commutators. Universality of conductance arises as a natural consequence of the nonrenormalization of anomalies. To illustrate our formalism we examine transport properties of a quantum wire in $(1+1)$ dimensions and of massless QED in background magnetic field in $(3+1)$ dimensions.

PACS numbers: 05.60.+w, 11.40.Dw, 73.23.Ad, 11.15.Ex
\end{abstract}

Transport properties of a physical system are usually linked to complicated dynamical processes (such as impurity scattering, inter-particle interactions etc.) and, in general, are not universal. Systems not exhibiting any dissipative processes may, however, exhibit some universal transport coefficients that are insensitive to changes in the microscopic constitution of the system. When one encounters a universal transport coefficient one should look for a physical principle explaining it's universality. In the quantum Hall effect, for instance, the universality of the Hall conductance can be linked to gauge invariance [2]. Another example is superconductivity, where it is the spontaneous breaking of the $U(1)$ gauge symmetry [1] that leads to the vanishing of the longitudinal resistivity and to the Meissner effect. In both examples the conductivity is universal with amazing accuracy, which is the result of the existence of a gap in the spectrum of bulk charged excitations. Note, however, that an incompressible quantum Hall system with an edge must support a branch of compressible edge states, which play essential role in understanding Hall quantization.

In this paper we consider a class of physical systems having no gap for charged excitations, yet exhibiting universal transport properties. At low energies these systems are assumed to possess two commuting conserved chiral charges $Q_{L}$ and $Q_{R}$ corresponding to the particles of left and right chirality. Although our method is not limited to the electric transport, we consider the electric conductance as a representative example. The latter is defined as $G=I / V$, where $I$ is the electric current and $e V=\mu_{L}-\mu_{R}$ is the difference between the chemical potentials $\mu_{L}$ and $\mu_{R}$ of the reservoirs of the particles of left and right chirality. We show that the conductance $G$ is independent of the dynamics of the system as long as the charges $Q_{L}$ and $Q_{R}$ are conserved. The principle that protects the universality is the nonrenormalization of chiral anomalies by interactions [3]. To illustrate our conclusions we examine the transport properties of a quantum wire in $(1+1)$ dimensions and of massless QED in a background magnetic field in $(3+1)$ dimensions.
The main tool we are using is a new formula (8) which relates the DC current to the anomalous commutators. In order to obtain this formula we use the methods of equilibrium statistical mechanics - an approach that has proven to be effective at the description of some transport phenomena in solids [ 6 . The only condition imposed on the dynamics of the system is the existence of two commuting conserved charges $Q_{L}$ and $Q_{R}$.

$$
\left[\mathcal{H}, Q_{L}\right]=\left[\mathcal{H}, Q_{R}\right]=0,\left[Q_{L}, Q_{R}\right]=0 .
$$

Here $\mathcal{H}$ is the Hamiltonian of the theory. We denote the conserved Noether currents corresponding to the charges $Q_{L}$ and $Q_{R}$ by $j_{L}^{\mu}$ and $j_{R}^{\mu}$

$$
\partial_{\mu} j_{L}^{\mu}=0, \partial_{\mu} j_{R}^{\mu}=0 .
$$

In the examples below $j_{L}$ and $j_{R}$ are chiral currents corresponding to the fermions of left and right chirality (in $1+1$ or in $3+1$ dimensions). The observable we are interested in is the electric current $j_{e}^{\mu}=e\left(j_{L}^{\mu}+j_{R}^{\mu}\right)$ ( $e$ is the elementary electric charge). Henceforth, we will refer to the difference $j_{a}^{\mu}=e\left(j_{L}^{\mu}-j_{R}^{\mu}\right)$ as to the axial current.

Physically, the conservation of the charges $Q_{L}$ and $Q_{R}$ means that there is no scattering of the particles of left chirality to the right ones and vice versa.

The conserved charges $Q_{L}$ and $Q_{R}$ are conjugate to the chemical potentials $\mu_{L}$ and $\mu_{R}$ of the external reservoirs of the particles of left and right chirality. The thermal state of the system connected to the external reservoirs is given by the density matrix

$$
\Sigma_{\mu}=e^{-\beta \mathcal{H}_{\mu}}, \mathcal{H}_{\mu}=\mathcal{H}+\mu_{L} Q_{L}+\mu_{R} Q_{R}
$$

and its transport properties are described by equilibrium statistical mechanics.

The continuity equation for the electric current $j_{e}^{\mu}=$ $(\rho, \mathbf{j})$ reads

$$
\partial_{\mu} j_{e}^{\mu}=0
$$

In $(d+1)$ dimensions it can be solved in terms of an antisymmetric tensor field $b$ of rank $d-1$ : 


$$
j_{e}^{\mu}=\epsilon^{\mu \nu_{1} \ldots \nu_{d}} \partial_{\nu_{1}} b_{\nu_{2} \ldots \nu_{d}},
$$

where $\epsilon^{\mu \nu_{1} \ldots \nu_{d}}$ is Levi-Civita's antisymmetric tensor. Note, that the field $b$ is not a physical observable of the system. A shift $b \mapsto b+h$, where the tensor $h$ satisfies $\epsilon^{\mu \nu_{1} \ldots \nu_{d}} \partial_{\nu_{1}} h_{\nu_{2} \ldots \nu_{d}}=0$, does not change the physical quantities $j^{\mu}$ and can be interpreted as a gauge transformation. This gauge freedom results in the field $b$ only having $d$ physical degrees of freedom. One may choose a specific gauge for the potential $b$ which allows to explicitly express the physical degrees of freedom of the field $b$ in terms of a d-vector field $\mathbf{a}$ :

$$
\rho=e \nabla \cdot \mathbf{a}, \mathbf{j}=-e \partial_{t} \mathbf{a} .
$$

In the equilibrium state characterized by the chemical potentials $\mu_{L}$ and $\mu_{R}$ the expectation value of the current is given by

$$
\begin{array}{r}
\langle\mathbf{j}(x)\rangle_{\mu}=-e\left\langle\partial_{t} \mathbf{a}(x)\right\rangle_{\mu}=\frac{i e}{\hbar}\langle[\mathcal{H}, \mathbf{a}(x)]\rangle_{\mu}= \\
=\frac{i e}{\hbar} Z_{\mu}^{-1} \operatorname{Tr}\left(e^{-\beta H_{\mu}}\left[\mathcal{H}_{\mu}, \mathbf{a}(x)\right]\right)- \\
-\frac{i e}{\hbar} Z_{\mu}^{-1} \operatorname{Tr}\left(e^{-\beta H_{\mu}}\left[\mu_{L} Q_{L}+\mu_{R} Q_{R}, \mathbf{a}(x)\right]\right),
\end{array}
$$

where $Z_{\mu}=\operatorname{Tr} \Sigma_{\mu}$. At first sight both terms on the r.h.s. of (7) must vanish by cyclicity of the trace, because $\mathcal{H}_{\mu}$ and $\mu_{L} Q_{L}+\mu_{R} Q_{R}$ commute with $\exp \left(-\beta \mathcal{H}_{\mu}\right)$. However, we are not allowed to use the formula $\operatorname{Tr}[a, b] c=$ $\operatorname{Tr}(a b c)-\operatorname{Tr}(b a c)$, because the triple products are too singular (not of trace class).

A more careful analysis shows that only the first trace on the r.h.s of (7) vanishes. Indeed, if we regularize the system by modifying the Hamiltonian $\mathcal{H}$ in such a way that a small spectral gap above the ground state energy is opened then, for a system in a finite box, $e^{-\beta \mathcal{H}_{\mu}}$ is of trace class, and the appropriately smeared field $\mathbf{a}(x)$ is bounded by some function of $\mathcal{H}_{\mu}$. Then the first trace on the l.h.s. of (7) vanishes, by cyclicity of the trace. But this argument cannot be applied to the second trace on the r.h.s. of (『) , because, after regularization, $Q_{L}$ and $Q_{R}$ do no longer commute with $\mathcal{H}_{\mu}$.

Finally, formula (7) yields the universal result

$$
\begin{array}{r}
\langle\mathbf{j}(x)\rangle_{\mu}=-\frac{i e}{\hbar}\left\langle\left[\mu_{L} Q_{L}+\mu_{R} Q_{R}, \mathbf{a}(x)\right]\right\rangle_{\mu}= \\
-\frac{i}{2 \hbar}\left(\mu_{L}-\mu_{R}\right) \int d y\left\langle\left[j_{a}^{0}(y), \mathbf{a}(x)\right]\right\rangle_{\mu} .
\end{array}
$$

Formula (81) expresses the electric current in terms of the anomalous commutator of the time component of the axial current with the field a solving the continuity equation.

Next, we want to show how nontrivial physical conclusions can be arrived at by applying formula (8) to concrete physical systems. Our first example is a onedimensional interacting electron liquid (quantum wire). It has long been understood [ [4] that the conductance
$G=I / V$ (where $I$ is the electric current and $V$ is the voltage drop) of a pure quasi one-dimensional electron system must be quantized in units of $2 e^{2} / h$ [ [ $[$ ], i.e.,

$$
G=2 n \frac{e^{2}}{h}, n=0,1,2, \ldots
$$

where $e$ is the elementary electric charge and $h$ is Planck's constant. The factor of 2 on the r.h.s. of (9) originates in the spin of electrons, the factor of $n$ corresponds to the number of filled energy bands of transversal quantization which form one-dimensional conducting channels.

It was far from clear, however, how the electronelectron interaction influenced the conductance. For a long time it was believed that repulsive electron-electron interactions should suppress the conductance. Recent experiments [7] showed that the quantization formula (9) holds true independently of the strength of electronelectron interactions in the wire as long as scattering off impurities is negligible and the voltage drop $V$ is not very large.

It was argued in [8] that the nonrenormalization of conductance by electron-electron interactions was due to the strong influence of the boundary conditions imposed by the reservoirs. This idea was supported by calculating the current-current correlation function of a model one-dimensional system where the reservoirs were modeled by turning off electron-electron interactions outside some finite region of the system. Conductance quantization in quantum wires and Quantum Hall systems has been compared in [6].

We shall show that a pure quantum wire (with no impurity backscattering) satisfies the dynamical requirement (11) and the universality of conductance quantization, as expressed in Eq. (9), follows directly from formula (8). For simplicity, we shall consider spinless fermions and drop the factor of 2 in (9).

The Hamiltonian of a general one-dimensional interacting fermionic system is given by

$$
\mathcal{H}=i \hbar v_{F} \int d x\left(\psi_{L}^{*} \partial_{x} \psi_{L}-\psi_{R}^{*} \partial_{x} \psi_{R}\right)+\mathcal{H}_{i n t},
$$

where $\psi_{L}$ and $\psi_{R}$ are left- and right-moving electrons of the noninteracting model, and $\mathcal{H}_{\text {int }}$ is the interaction Hamiltonian. It includes higher order terms in $\psi$ corresponding to electron-electron scattering as well as quadratic terms responsible, for nonlinearity of dispersion. We refer to modes created by $\psi_{L}^{*}$ and $\psi_{R}^{*}$ as to leftand right-movers in spite of the fact that dynamically they are not necessarily quasi-particles of the interacting model. One can introduce densities of left- and rightmovers $\psi_{L}^{*} \psi_{L}=n_{L}, \psi_{R}^{*} \psi_{R}=n_{R}$. The total charge density is given by $\rho=e\left(n_{L}+n_{R}\right)$. The expression for the electric current density $j$ in terms of $\psi_{L}$ and $\psi_{R}$ is not universal and depends on the particular form of $\mathcal{H}_{\text {int }}$.

If we assume that the junctions between the onedimensional system and the electron reservoirs are adiabatic, the conserved charges conjugate to the chemical 
potentials of the reservoirs are equal to the integrals of $n_{L}$ and $n_{R}$ :

$$
Q_{L}=\int d x n_{L}, Q_{R}=\int d x n_{R}
$$

We assume that these charges commute with the interacting Hamiltonian (10).

It is convenient to use one-dimensional bosonization formulae for the Fermi fields $\psi_{L}$ and $\psi_{R}$.

$$
\begin{aligned}
& \psi_{L}^{*}=e^{2 \pi i \phi_{L}}, \psi_{L}=e^{-2 \pi i \phi_{L}} \\
& \psi_{R}^{*}=e^{-2 \pi i \phi_{R}}, \psi_{R}=e^{2 \pi i \phi_{R}} .
\end{aligned}
$$

The bosonic fields $\phi_{L}$ and $\phi_{R}$ satisfy the commutation relations

$$
\begin{array}{r}
{\left[\phi_{L, R}(x), \phi_{L, R}(y)\right]= \pm \frac{i}{4 \pi} \varepsilon(x-y),} \\
{\left[\phi_{L}(x), \phi_{R}(y)\right]=\frac{i}{4 \pi},}
\end{array}
$$

where $\varepsilon(x-y)=1, x>y$; and $\varepsilon(x-y)=-1, x<y$. The densities of left- and right-moving particles acquire the form $n_{L}=\partial_{x} \phi_{L}, n_{R}=\partial_{x} \phi_{R}$. The conserved charges $Q_{L}$ and $Q_{R}$ have the following commutation relations with the bosonic fields:

$$
\left[Q_{L}, \phi_{L}(x)\right]=\frac{i}{2 \pi},\left[Q_{R}, \phi_{R}(y)\right]=-\frac{i}{2 \pi} .
$$

The electric charge density is then given by

$$
\rho=e\left(\partial_{x} \phi_{L}+\partial_{x} \phi_{R}\right)=e \partial_{x} a,
$$

where $a$ is the current potential $a=\phi_{L}+\phi_{R}$. We note that all the commutation relations and bosonization rules listed above only depend on the kinematics of Fermi fields and are entirely independent of the dynamics of the system. Our only important dynamical assumption is the commutativity of the charges $Q_{L}$ and $Q_{R}$ with the Hamiltonian of the interacting system.

Formulae (8) and (14) can now be combined to yield the electrical conductance:

$$
\begin{gathered}
\langle j(x)\rangle_{\mu}=-i \frac{e}{\hbar}\left\langle\left[\mu_{L} Q_{L}+\mu_{R} Q_{R}, a(x)\right]\right\rangle_{\mu}= \\
=\frac{e}{h}\left(\mu_{L}-\mu_{R}\right) .
\end{gathered}
$$

This concludes the derivation of the universal conductance formula (9).

Next, we test formula (8) on a $(3+1)$-dimensional example of massless Dirac fermions coupled to the electromagnetic field. This system is described by the Lagrangian:

$$
\begin{aligned}
L=-\frac{1}{4} F^{\mu \nu} F_{\mu \nu}+ & \psi_{L}^{*} \sigma_{L}^{\mu}\left(i \hbar \partial_{\mu}-\frac{e}{c} A_{\mu}\right) \psi_{L}+ \\
& +\psi_{R}^{*} \sigma_{R}^{\mu}\left(i \hbar \partial_{\mu}-\frac{e}{c} A_{\mu}\right) \psi_{R}
\end{aligned}
$$

where $\sigma_{\mu}^{L}=\left(I, \sigma_{k}\right), \sigma_{\mu}^{R}=\left(I,-\sigma_{k}\right)$. Chiral currents $j_{L}^{\mu}=\psi_{L}^{*} \sigma_{\mu}^{L} \psi_{L}$ and $j_{R}^{\mu}=\psi_{R}^{*} \sigma_{\mu}^{R} \psi_{R}$ are not conserved because of the chiral anomaly. The conservation is recovered upon adding a Chern-Simons term [9]:

$$
\tilde{j}_{L, R}^{\mu}=j_{L, R}^{\mu} \pm \frac{\alpha^{2}}{8 \pi^{2} e^{2}} \epsilon^{\mu \nu \lambda \sigma} A_{\nu} \partial_{\lambda} A_{\sigma} .
$$

where $\alpha$ is the fine structure constant $\alpha=e^{2} / \hbar c$. The corresponding charges

$$
Q_{L}=\int d^{3} x \tilde{j}_{L}^{0}, Q_{R}=\int d^{3} x \tilde{j}_{R}^{0}
$$

being conserved, the conjugate chemical potentials $\mu_{L}$ and $\mu_{R}$ can be introduced. That $\mu_{R}$ is different from $\mu_{L}$ means that the average density of left-handed particles in the system is different from the average density of righthanded ones. Such a situation is encountered in some models of the early Universe 10 .

Our goal is to compute the expectation value of the electric current $\mathbf{j}$ in the background electromagnetic field $A_{\mu}$ applying formula (8):

$$
\langle\mathbf{j}(x)\rangle_{A}=-\frac{i e}{2 \hbar}\left(\mu_{L}-\mu_{R}\right)\left\langle\left[Q_{L}-Q_{R}, \mathbf{a}(x)\right]\right\rangle_{A} .
$$

The commutators of the densities of the left- and the right-handed fermions are given by [9]:

$$
\left[\tilde{j}_{L, R}^{0}(x), \tilde{j}_{L, R}^{0}(y)\right]= \pm \frac{i \alpha}{4 \pi^{2} e} \partial_{k}\left(B_{k}(x) \delta(x-y)\right)
$$

whereas the commutator of the left-handed and the righthanded current is zero. Here $B_{k}=\epsilon^{i j k} \partial_{i} A_{j}$ is the magnetic field strength. The commutator of axial and electric charge densities is of the form:

$$
\left[\rho_{a}(x), \rho_{e}(y)\right]=\frac{i e \alpha}{2 \pi^{2}} \partial_{k}\left(B_{k}(x) \delta(x-y)\right) .
$$

Assuming that the commutator of $\rho_{a}$ with a is local, one can remove the divergence on the r.h.s of (22):

$$
\left[\rho_{a}(x), a_{k}(y)\right]=\frac{i \alpha}{2 \pi^{2}} B_{k}(x) \delta(x-y)+\ldots
$$

where the ... stand for a term of the form of a curl of some vector field. Substituting (23) into 20 yields

$$
\left\langle j_{k}(x)\right\rangle_{A}=\frac{\alpha}{4 \pi^{2} \hbar}\left(\mu_{L}-\mu_{R}\right) B_{k}(x) .
$$

Note that only the first term on the r.h.s. of (23) contributes to the current. Another one drops out due to the integration in (19). The result (24) can be easily verified on the example of a non-interacting system in a constant uniform magnetic field, where the single-particle picture of [4] can be implemented. Our derivation implies that formula (24) holds true when the magnetic field is not necessarily uniform. In analogue to the previously considered quantum wire the formula for the DC current 
(24) is not affected by interactions preserving charges $Q_{L}$ and $Q_{R}$ [3]. Coupling to a $U(1)$ gauge field in (17) is an example of such an interaction.

Our next goal is to exhibit a relation between the conductance formula (8) and the Goldstone theorem. Recall that the Goldstone theorem states that in a system with spontaneous symmetry breaking there is a massless mode (Goldstone boson). At zero temperature the usual proof proceeds as follows (see e.g. [11]): assume that the symmetry group is compact, and denote the symmetry generators by $L^{a}$,

$$
L^{a}=\int d x j^{a}(x)
$$

where $j^{a}$ stand for time components of conserved Noether currents.

Spontaneous symmetry breaking manifests itself in a nonvanishing expectation value

$$
\left\langle\left[L^{a}, \Phi(x)\right]\right\rangle \neq 0
$$

of a commutator of a symmetry generator $L^{a}$ with some operator $\Phi$. By eq. (25), (26) implies that

$$
\left(\int_{t=s+\epsilon} d y-\int_{t=s-\epsilon} d y\right)\left\langle T\left(j^{a}(t, y) \Phi(s, x)\right)\right\rangle \neq 0 .
$$

Since the current $j_{\nu}^{a}$ is conserved, the integration surface can be deformed into a sphere of arbitrarily large radius $R$. In order to obtain a nonvanishing expectation value in (27), the correlation function of $j^{a}(y)$ and $\Phi(x)$ must decay as $R^{-d}$, where $d$ is the spatial dimension. This implies the existence of massless modes in the system.

Note that the r.h.s. of the universal conductance formula (8) is exactly of the form (27). The Noether current in this problem is the axial current $j_{a}(x)$, the symmetry group is the axial symmetry. Like in the derivation of the Goldstone theorem, a nonvanishing expectation value of the current, $\langle j(x)\rangle_{\mu} \neq 0$, implies the existence of a massless mode in the system. For instance, in the one dimensional transport problem this gapless mode is the density wave described by the field $a(x)$. The existence of this mode implies that, in the limit of large distance and low frequency scales, the system is described by a conformal field theory with a chiral algebra which contains a $U(1)$ current algebra. In our simple example this conformal field theory is described by the Luttinger model.

Although we observe a close analogy between the derivation of the Goldstone theorem and our formula (8), there is an important physical difference. It is best illustrated by working out the example of the quantum wire. There is, in fact, no spontaneous symmetry breaking in the one-dimensional transport problem. The axial symmetry group of this system is $U(1)$. When one introduces an operator $a(x)$ solving the continuity equation, one decompactifies the axial group from $U(1)$ to $R$. Indeed, under the action of the symmetry generator $Q_{L}-Q_{R}$ the field $a(x)$ is shifted by a constant,

$$
\left[Q_{L}-Q_{R}, a(x)\right]=\frac{i}{\pi}
$$

The group $U(1)$ has no representations of this type, a constant cannot be in the same multiplet as a nontrivial field. By introducing the unphysical field $a(x)$ we effectively replace $U(1)$ by its covering group $R$. Of course, it is not surprising to find that the expectation value of a constant is nonvanishing. But this fact is not related to any physical symmetry breaking. Note, that this situation is special for abelian symmetry groups.

Acknowledgements. A.A. and V.C. are grateful to ETH, Zürich for hospitality during the period when this paper was written. We thank J.Adams and J.Mickelsson for useful discussions.

[1] V.L.Ginzburg and L.D.Landau, Zh. Eksp. Teor. Fiz. 20, 1064 (1950);

[2] R.B.Laughlin, Phys. Rev B 235632 (1981); B.I.Halperin, Phys. Rev. B 252185 (1982).

[3] S.Adler, Phys. Rev. 177, 2426 (1969); S.Adler and W.Bardeen, Phys. Rev. 182, 1517 (1969).

[4] R.Landauer, Philos. Mag. 21863 (1970); M.Büttiker, Phys. Rev. Lett. 57, 1761 (1986).

[5] Y. Oreg and A. Finkel'sten, Phys. Rev. Lett. 74, 3668 (1995).

[6] A.Yu.Alekseev, V.V.Cheianov and J.Fröhlich, Phys. Rev. B 54, R17320 (1996).

[7] B.J. van Wees et al., Phys. Rev. Lett. 60, 848 (1988); S.Tarucha, T.Honda, and T.Saku, Solid State Commun. 94, 413 (1995);

A.Yacoby et al. Phys. Rev. Lett. 77, 4612 (1996).

[8] D.L.Maslov and M.Stone, Phys. Rev. B 52, R5539 (1995);

V.V.Ponomarenko, Phys. Rev. B 52, R8666 (1995); I.Safi and H.J.Schulz, Phys. Rev. B 52, R17040 (1995); A.Kawabata, J. Phys. Soc. Japan 65, 30 (1996).

[9] See the article by R. Jackiw in S.B.Treiman, R.Jackiw, B.Zumino and E.Witten, Current Algebras and Anomalies, World Scientific (1985).

[10] J.A.Harvey and M.S.Turner, Phys. Rev. D 42, 3344 (1990).

[11] C.Itzykson, J.-B.Zuber, Quantum Field Theory, International Series in Pure and Applied Physics, McGraw-Hill Inc. (1980) 519. 